\title{
Influence du diabète antérieur à la grossesse sur les anomalies congénitales au Canada entre 2002 et 2012
}

\author{
S. Liu, M.B., Ph. D. (1); J. Rouleau (1); J. A. León, M.D. (1); R. Sauve, M.D. (2); K. S. Joseph, M.D., Ph. D. (3, 4, 5); \\ J. G. Ray, M.D. (6); Système canadien de surveillance périnatale (1)
}

Cet article a fait l'objet d'une évaluation par les pairs.

\section{Résumé}

Objectif : Examiner l’influence du diabète antérieur à la grossesse sur la prévalence à la naissance des anomalies congénitales au Canada.

Méthodologie : Nous avons réalisé une étude en population chez l'ensemble des femmes ayant accouché à l'hôpital au Canada (hors Québec) entre avril 2002 et mars 2013, ainsi que chez leurs enfants nés vivants ayant un poids à la naissance d'au moins 500 grammes ou un âge gestationnel d'au moins 22 semaines. On a attribué les codes de diagnostic de la CIM-10 au diabète de type 1 ou de type 2 antérieur à la grossesse. L'association entre le diabète et l'ensemble des anomalies congénitales ainsi qu'avec les diverses catégories prises séparément a été estimée à l'aide des rapports de cotes ajustés et son influence a été calculée sous forme de pourcentage de risque attribuable dans la population (\%RAP).

Résultats : Sur les 2839680 naissances vivantes, 118892 nouveau-nés étaient atteints d'anomalies congénitales (41,9 pour 1 000). Même si la prévalence des anomalies congénitales est passée de 50,7 pour 1000 naissances vivantes en 2002-2003 à 41,5 pour 1000 en 2012-2013, le \%RAP d'une anomalie congénitale liée au diabète antérieur à la grossesse est passé de 0,6\% (intervalle de confiance [IC] à $95 \%: 0,4$ à 0,8) à 1,2\% (IC à $95 \%: 0,9$ à 1,4). Plus précisément, le \%RAP des cardiopathies congénitales est passé de $2,3 \%$ (IC à $95 \%: 1,7$ à 2,9) à 4,2\% (IC à $95 \%: 3,5$ à 4,9) et celui des anomalies gastrointestinales de $0,8 \%$ (IC à $95 \%: 0,2$ à 1,9) à $1,4 \%$ (IC à $95 \%: 0,7$ à 2,6) au cours de la période étudiée.

Conclusion : En dépit de la diminution relative de la prévalence des anomalies congénitales au Canada, la proportion d'anomalies congénitales pouvant être attribuées au diabète maternel antérieur à la grossesse a augmenté. On recommande l'amélioration des initiatives en matière de soins préconceptionnels destinées aux femmes diabétiques.

Mots-clés : diabète, antérieur à la grossesse, anomalie congénitale, malformation congénitale, tendance, pourcentage de risque attribuable dans la population

\section{Introduction}

Les anomalies congénitales majeures touchent environ $3 \%$ à $5 \%$ des nouveau-nés au Canada et elles constituent la principale cause de mortalité périnatale et infantile ${ }^{1-3}$. Les anomalies congénitales peuvent également nécessiter des interventions chirurgicales et des soins particuliers pendant plusieurs années après la naissance ${ }^{4}$.
Diffuser cet article sur Twitter

Principales constatations

- Le diabète antérieur à la grossesse a presque doublé chez les Canadiennes de 35 ans et plus, passant de 6,4 par 1000 naissances vivantes en 2002 à 12,3 par 1000 naissances vivantes en 2012.

- La proportion d'anomalies congénitales attribuables à un diabète de la mère antérieur à sa grossesse a augmenté depuis 10 ans.

- $2,3 \%$ des cardiopathies congénitales sont attribuables à un diabète antérieur à la grossesse en 2002, contre $4,2 \%$ en 2012 .

- Une hausse des proportions des anomalies congénitales gastro-intestinales, génito-urinaires et musculosquelettiques est également attribuable à un diabète antérieur à la grossesse.

Les enfants de femmes diabétiques de type 1 et de type 2 avant la grossesse sont de 2 à 10 fois plus susceptibles que les enfants de mères non diabétiques d'être atteints d'anomalies congénitales, en particulier de cardiopathies ou d'anomalies du système nerveux cen$\operatorname{tral}^{5-11}$. Comme la fréquence du diabète antérieur à la grossesse a presque doublé en dix ans ${ }^{12}$, il serait avantageux pour les programmes en pratique clinique et en santé publique de savoir si l'effet du diabète antérieur à la grossesse sur la fréquence des anomalies congénitales a également changé.

Rattachement des auteurs :

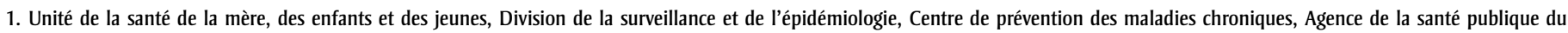
Canada, Ottawa (Ontario), Canada

2. Départements de pédiatrie et des sciences de la santé communautaire, Université de Calgary, Calgary (Alberta), Canada

3. Département d'obstétrique et de gynécologie, Université de la Colombie-Britannique, Vancouver (Colombie-Britannique), Canada

4. Children's and Women's Hospital de la Colombie-Britannique, Vancouver (Colombie-Britannique), Canada

5. École de santé publique et de santé des populations, Université de la Colombie-Britannique, Vancouver (Colombie-Britannique), Canada

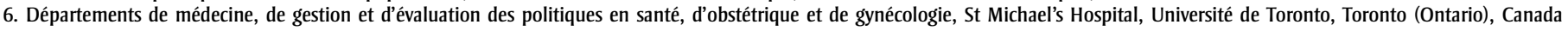

Correspondance : Shiliang Liu, bureau 405A2, I.A. 6804A, 785, avenue Carling, Ottawa (Ontario) K1A 0K9; courriel : shiliang.liu@phac-aspc.gc.ca 
Des soins préconceptionnels sont recommandés pour les femmes atteintes de diabète. Ils comprennent, pendant la période périconceptionnelle, la maîtrise de la glycémie, un apport nutritionnel optimal et une supplémentation en acide folique ${ }^{13}$. En outre, un diagnostic prénatal précoce des anomalies congénitales létales et les interruptions de grossesse qui en résultent jouent un rôle important dans la gestion de cette grossesse chez les femmes diabétiques avant leur grossesse ${ }^{14}$.

Dans le cadre de cette étude, nous avons examiné l'impact du diabète antérieur à la grossesse sur la prévalence à la naissance des anomalies congénitales au Canada. Plus précisément, nous avons utilisé le pourcentage de risque attribuable dans la population (\% RAP). Cet indice quantifie la proportion d'anomalies congénitales attribuables au diabète antérieur à la grossesse qui pourraient être prévenues en optimisant la maîtrise du diabète.

\section{Méthodologie}

Nous avons effectué une étude de cohorte sur l'ensemble des naissances vivantes au Canada (hors Québec) entre 2002-2003 et 2012-2013. Nous avons recueilli les données sur chaque naissance en milieu hospitalier dans la Base de données sur les congés des patients (BDCP) de l'Institut canadien d'information sur la santé (ICIS). La BDCP contient les données résumées et colligées pour chaque hospitalisation au Canada hors Québec. Ces données sont extraites par des archivistes médicaux formés dans chaque hôpital et codées conformément à un protocole uniforme. Les dossiers d'hospitalisation pour l'accouchement de la mère et la naissance du nouveau-né vivant ont été reliés au moyen d'un numéro commun à la mère et au nouveau-né. Le fichier de la BDCP contient des données individuelles et des renseignements sur l'utilisation des services de santé, les diagnostics et les interventions. Les données de la BDCP ont déjà été validées ${ }^{15}$ et elles sont très utilisées à des fins de recherche $e^{6,16,17}$ ainsi que dans un grand nombre de rapports sur la surveillance périnatale au Canada ${ }^{18}$, notamment des rapports sur la surveillance des anomalies congénitales ${ }^{1}$.
Nous avons inclus toutes les paires mère/ nouveau-né, à l'exception des naissances survenues à 22 semaines de gestation ou moins et des nouveau-nés qui avaient un poids à la naissance inférieur à 500 grammes. Les variables utilisées dans cette étude sont la province de résidence de la mère, le code postal à trois caractères, le code de résidence, l'année de l'accouchement, la parité, le sexe du nouveau-né, les dates d'admission et de congé pour l'accouchement, la date de décès du nouveau-né (le cas échéant), le poids du nouveau-né à la naissance ainsi que l'issue de l'accouchement (naissance vivante ou mortinaissance). Toutefois, les enfants mortnés n'ont pas été inclus dans la population étudiée car leurs dossiers ne pouvaient pas être liés au dossier de la mère. Le diabète antérieur à la grossesse (de type 1 ou de type 2) et les anomalies congénitales ont été repérés d'après les données des 25 champs de diagnostic du dossier médical codés selon la Classification statistique internationale des maladies et des problèmes de santé connexes, dixième révision, Canada (CIM-10-CA). Le diagnostic d'une anomalie congénitale est établi d'après le dossier lié à l'hospitalisation à la naissance ou à une réadmission à l'hôpital dans les 30 jours suivant la naissance $^{1,18}$. Pour la catégorisation des anomalies congénitales dans la BDCP, nous avons regroupé en un seul enregistrement toutes les admissions du même nourrisson afin d'éviter les doublons ${ }^{1}$. Nous avons produit un résumé en fonction des codes individuels de la CIM-10, de 59 catégories standard et de 14 catégories plus larges (c.-à-d. combinant quelques catégories d'anomalies congénitales). Les catégories d'anomalies congénitales ont été définies par une liste d'au moins un code diagnostic de la CIM-10, chaque catégorie comprenant tous les nouveau-nés auxquels a été attribué au moins un des codes associés à la catégorie. Par exemple, un nouveau-né présentant une malformation des membres supérieurs et une malformation des membres inférieurs constitue un cas de malformation des membres. Toutefois, les anomalies des membres supérieurs et celles des membres inférieurs peuvent faire partie de deux catégories distinctes car elles ont chacune leur propre code de la CIM-10-CA. Par ailleurs, les nouveau-nés atteints de plusieurs anomalies contenues dans différentes catégories sont comptabilisés dans chacune des catégories.
Nous avons calculé la prévalence de l'ensemble des anomalies congénitales et la prévalence par catégorie pertinente pour l'analyse. Par la suite, nous avons déterminé la prévalence des anomalies congénitales associées aux femmes atteintes de diabète antérieurement à leur grossesse. Les rapports de cotes (RC) bruts et ajustés ainsi que les intervalles de confiance (IC) à $95 \%$ ont été estimés pour l'association entre diabète antérieur à la grossesse et anomalies congénitales. Nous avons utilisé une régression logistique multivariée ajustée pour l'âge maternel (moins de 20 ans, 20 à 24 ans, 25 à 29 ans, 30 à 34 ans, 35 à 39 ans et 40 ans et plus), la parité (c.-à-d. le nombre de naissances précédentes, soit $0,1,2,3$ ou plus, inconnu) et l'année de l'accouchement. Nous avons également estimé, à l'aide de l'équation ci-dessous, la proportion d'anomalies congénitales éliminées si un facteur de risque comme le diabète antérieur à la grossesse (qu'on estime être associé de manière causale aux anomalies congénitales) était supprimé de la population, afin de mesurer l'effet ajusté :

$$
\operatorname{pd}(\mathrm{RC}-1) / \mathrm{RC} \text {, }
$$

où pd représente la proportion de cas exposés au facteur de risque et $\mathrm{RC}$, le rapport de cotes ajusté attribuable au facteur de risque $^{19}$. Un RC ajusté représentant la relation entre le diabète antérieur à la grossesse et les anomalies congénitales a été calculé pour chaque année. Dans la mesure où la prévalence varie, le diabète antérieur à la grossesse peut avoir entraîné des modifications de la force de la relation entre diabète antérieur à la grossesse et anomalies congénitales. Toutes les analyses statistiques ont été réalisées à l'aide de la version $9.2 \mathrm{du}$ logiciel SAS pour Unix (SAS Institute, Cary, Caroline du Nord, États-Unis).

Cette étude a été réalisée sous l'égide de l'Agence de la santé publique du Canada, qui a le mandat fédéral d'assurer le suivi de la santé de la population canadienne. Les données analysées ayant été anonymisées, l'approbation d'un comité d'éthique n'était pas nécessaire.

\section{Résultats}

La population à l'étude est de 2839680 naissances vivantes. La prévalence du 
diabète antérieur à la grossesse chez les mères de nouveau-nés vivants a augmenté significativement au cours des 11 années de l'étude, passant de 4,6 pour 1000 naissances vivantes en 2002-2003 à 7,5 pour 1000 naissances vivantes en 2012-2013. La prévalence du diabète de type 1 chez les mères de nouveau-nés vivants est restée stable, soit 2,7 pour 1000 naissances vivantes en 2002-2003 et 2,8 pour 1000 naissances vivantes en 2012-2013 (valeur $p$ de la tendance linéaire : 0,43), alors que la prévalence du diabète maternel de type 2 est passée de 1,9 pour 1000 naissances vivantes à 4,7 pour 1000 naissances vivantes en 20122013 (valeur $p$ de la tendance linéaire inférieure à 0,0001). Chez les femmes de 35 ans ou plus ayant donné naissance à un enfant vivant, la fréquence du diabète antérieur à la grossesse a globalement presque doublé, passant de 6,4 pour 1000 naissances vivantes à 12,3 pour 1000 entre 2002-2003 et 2012-2013 (figure 1).

$\mathrm{Au}$ total, 118892 nouveau-nés atteints d'au moins une anomalie congénitale ont été repérés parmi les 2839680 nourrissons nés pendant la période à l'étude, ce qui représente 41,9 anomalies congénitales pour 1000 naissances vivantes. La prévalence des anomalies congénitales a baissé entre 2002-2003 et 2007-2008, passant de 50,7 pour 1000 à 38,5 pour 1000 naissances vivantes, puis est remontée à 41,5 pour 1000 en 2012-2013. La prévalence de la majorité des catégories plus larges d'anomalies a diminué pendant l'ensemble de la période, par exemple celle des anomalies musculosquelettiques et cardiovasculaires, alors que la prévalence des anomalies génito-urinaires a augmenté (figure 2).

La fréquence des anomalies congénitales était de 93,4 pour 1000 naissances vivantes chez les femmes atteintes de diabète antérieurement à leur grossesse contre 41,5 pour 1000 naissances vivantes chez les femmes non diabétiques avant leur grossesse. La fréquence était semblable pour le diabète de type 1 (93,7 pour 1000 naissances vivantes) et le diabète de type 2 (93,3 pour 1000 naissances vivantes). Le RC ajusté pour les anomalies congénitales associées au
FIGURE 1

Tendances du diabète antérieur à la grossesse selon l'âge de la mère, Canada (hors Québec), 2002-2003 à 2012-2013

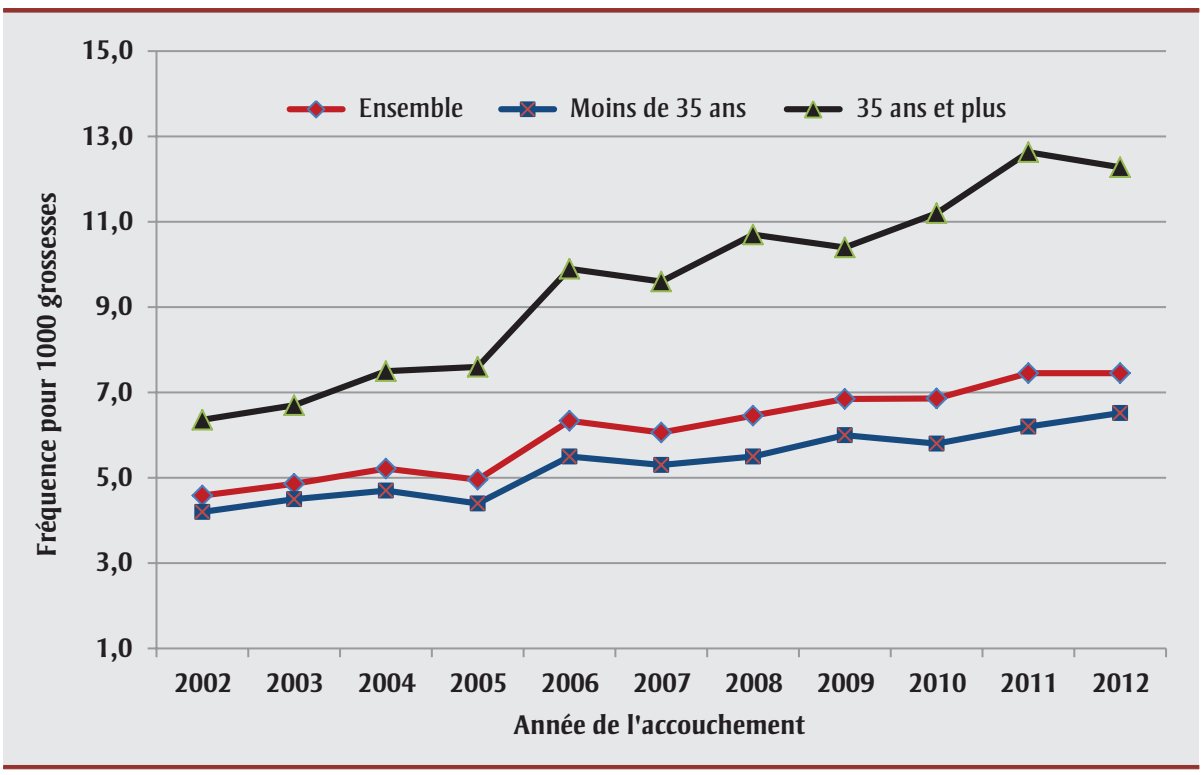

diabète de type 1 pour l'ensemble de la population était de 2,38 (IC à $95 \%: 2,20$ à 2,57$)$ alors que celui associé au diabète de type 2 était de 2,31 (IC à $95 \%: 2,16$ à 2,47). L'ampleur de L'association entre le diabète de type 1 et de type 2 et les catégories plus larges d'anomalies congénitales était semblable, sauf pour les

anomalies musculosquelettiques, qui n'étaient pas associées au diabète de type 1 (tableau 1).

Globalement, 0,9 \% des anomalies congénitales dans la population à l'étude étaient attribuables à un diabète antérieur à la grossesse (tableau 1). La contribution

FIGURE 2

Tendances de la prévalence à la naissance des trois principales catégories d'anomalies congénitales, Canada (hors Québec), 2002-2003 à 2012-2013

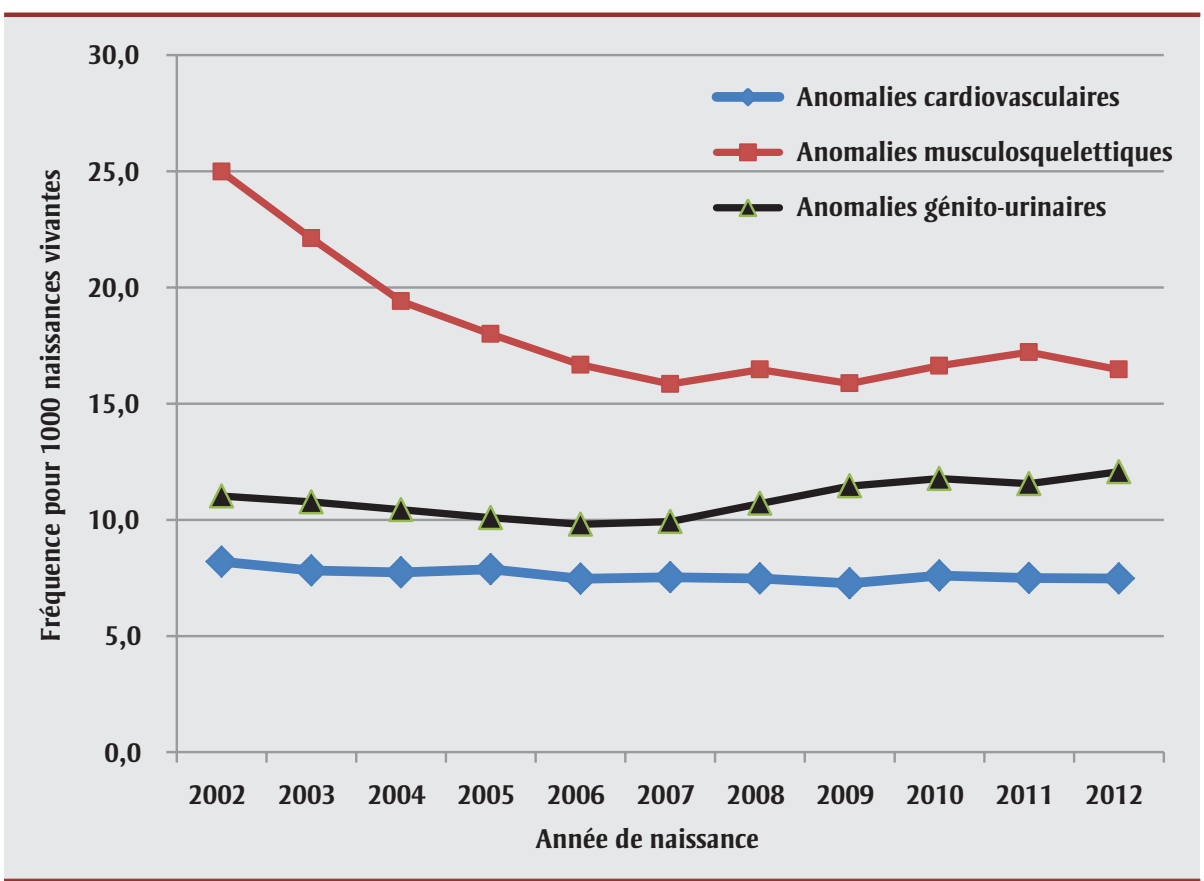


TABLEAU 1

Association entre le diabète de type 1 et de type 2 antérieur à la grossesse et les anomalies congénitales, Canada (hors Québec), $2002-2003$ à 2012-2013

\begin{tabular}{|c|c|c|c|c|c|c|c|}
\hline \multirow[t]{2}{*}{ Type d'anomalie } & \multicolumn{4}{|c|}{$\begin{array}{l}\text { Prévalence (pour } 1000 \text { naissances vivantes) } \\
\begin{array}{l}\text { Diabète } \\
\text { Sans diabète }\end{array}\end{array}$} & \multicolumn{2}{|c|}{$\begin{array}{l}\text { Rapport de cotes ajusté } \\
\text { (intervalle de confiance à } 95 \%)^{a}\end{array}$} & \multirow{2}{*}{$\begin{array}{c}\text { Pourcentage de } \\
\text { risque attribuable } \\
\text { dans la population (\%RAP) }\end{array}$} \\
\hline & Type 1 & Type 2 & Type 1 & Type 2 & Type 1 & Type 2 & \\
\hline Système nerveux central & 5,3 & 5,9 & 1,5 & 1,5 & 3,48 (3,55 à 4,76) & $3,85(2,97$ à 4,99$)$ & 1,66 \\
\hline Cardiovasculaire & 47,4 & 41,2 & 7,5 & 7,5 & $6,55(5,89$ à 7,29$)$ & $5,35(4,83$ à 5,89$)$ & 2,97 \\
\hline Fentes labio-palatines & 3,6 & 3,9 & 1,4 & 1,4 & $2,48(1,70$ à 3,63$)$ & $2,77(2,02$ à 3,80) & 1,03 \\
\hline Digestive & 4,7 & 4,0 & 1,6 & 1,6 & $3,06(2,20$ à 4,25$)$ & 2,41 (2,76 à 3,29) & 1,06 \\
\hline Musculosquelettique & 17,6 & 26,0 & 17,8 & 17,8 & $0,99(0,84$ à 1,18$)$ & $1,49(1,32$ à 1,69$)$ & 0,18 \\
\hline Ensemble des anomalies congénitales & 93,7 & 93,3 & 41,7 & 41,7 & $2,38(2,20$ à 2,57$)$ & 2,31 (2,16 à 2,47) & 0,85 \\
\hline
\end{tabular}

Abréviations : RAP, risque attribuable dans la population; RC, rapport de cotes.

Remarque : Le \%RAP est fondé sur le RC associé au diabète (de type 1 et de type 2).

${ }^{a}$ Ajusté pour l'âge maternel, le nombre d'accouchements précédents et l'année de naissance.

étiologique du diabète antérieur à la grossesse aux anomalies congénitales a augmenté entre 2002-2003 et 2012-2013 : le \%RAP annuel des anomalies congénitales associées au diabète antérieur à la grossesse a doublé pendant la période à l'étude, passant de 0,6\% (IC à $95 \%: 0,4$ à 0,8) en 2002-2003 à 1,2\% (IC à $95 \%: 0,9$ à 1,4) en 2012-2013 (figure 3). En effet, la prévalence du diabète maternel antérieur à la grossesse est passée de 4,6 pour 1000 naissances vivantes en 2002-2003 à 7,5 pour 1000 naissances vivantes en 2012-2013, alors que la relation entre

\section{FIGURE 3}

Tendances de la prévalence à la naissance de l'ensemble des anomalies congénitales et du pourcentage de risque attribuable dans la population (\%RAP) correspondant, Canada (hors Québec), 2002-2003 à 2012-2013

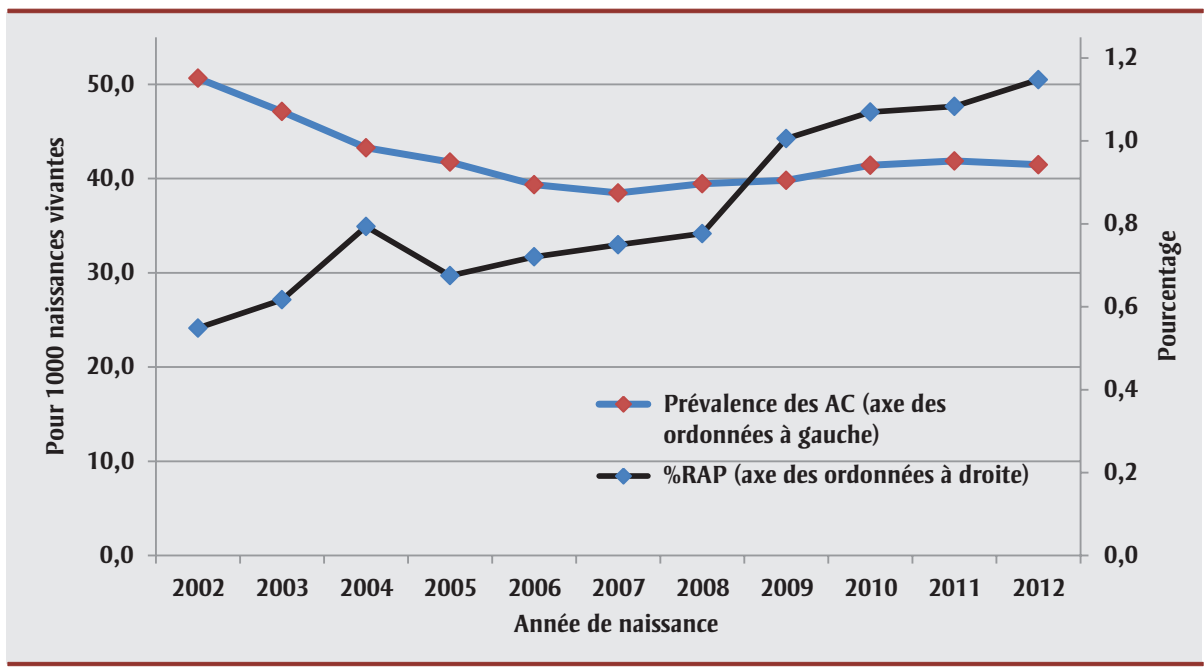

Abréviations : AC, anomalies congénitales; RAP, risque attribuable dans la population.
$3 \%$ des cardiopathies chez les nourrissons étaient attribuables à un diabète maternel antérieur à la grossesse (tableau 1). Le \% RAP correspondant a augmenté pendant la période à l'étude pour les anomalies cardiovasculaires, passant de 2,3\% (IC à $95 \%: 1,7$ à 2,9 ) à 4,2 \% (IC à $95 \%: 3,5$ à 4,9 ) et pour les anomalies gastro-intestinales, passant de $0,8 \%$ (IC à $95 \%: 0,2$ à 1,9 ) à $1,4 \%$ (IC à $95 \%: 0,7$ à 2,6), ainsi que pour les anomalies génito-urinaires et musculosquelettiques (figure 4).

\section{Analyse}

Au Canada, la prévalence à la naissance des anomalies congénitales était d'environ 42 pour 1000 naissances vivantes entre 20022003 et 2012-2013. La fréquence de plusieurs anomalies congénitales individuelles a diminué au cours de la période à l'étude. Toutefois, l'exposition des fœus au diabète maternel antérieur à la grossesse a augmenté pendant cette période, ce qui s'est traduit par une hausse de la contribution du diabète antérieur à la grossesse aux anomalies congénitales. Même si l'étiologie des anomalies congénitales est multifactorielle et largement inconnue $e^{1,4,20}$, plusieurs facteurs non génétiques jouent un rôle significatif dans leur apparition, en particulier l'âge, un diabète antérieur à la grossesse, l'obésité et une carence en acide folique chez la mère $e^{1,4,6,21-23}$. Notre analyse des données canadiennes récentes laisse penser qu'un diabète antérieur à la grossesse demeure un facteur de risque important et modifiable. 
FIGURE 4

Pourcentage de risque attribuable dans la population pour les anomalies congénitales associées aux femmes atteintes de diabète antérieur à la grossesse, Canada (hors Québec), comparaison entre la période 1 (années 2002-2003 et 2003-2004) et la période 2 (années 2011-2012 et 2012-2013)

\%RAP (IC à $95 \%)$

Ensemble des anomalies, période $1: 0,58(0,43$ à 0,74$)$

période 2 : $1,12(0,94$ à 1,31$)$

Système nerveux central, période $1: 1,67(0,80$ à 3,13$)$

période 2 : 1,77 (0,94 à 3,00)

Cardiovasculaire, période $1: 2,25(1,73$ à 2,89)

période 2 : 4,16 (3,53 à 4,89)

Fentes labio-palatines, période $1: 0,53(-0,02$ à 1,74$)$

période $2: 0,88(0,18$ à 2,11$)$

Gastro-intestinale, période 1 : $0,81(0,22$ à 1,90$)$

période 2 : $1,44(0,66$ à 2,62$)$

Génito-urinaire, période 1 : $0,43(0,18$ à 0,76$)$

période 2 : $0,74(0,47$ à 1,07$)$

Musculosquelettique, période $1: 0,05(-0,08$ à 0,23$)$

période 2 : $0,23(0,04$ à 0,45$)$

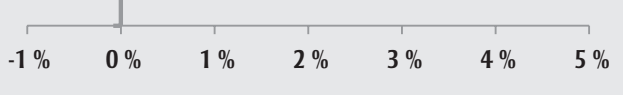

Abréviations : IC, intervalle de confiance; RAP, risque attribuable dans la population.

Remarque : Le \%RAP est calculé pour l'ensemble des anomalies congénitales et pour certaines catégories choisies à l'aide de l'équation décrite à la section Méthodologie (pd $(\mathrm{RC}-1) / \mathrm{RC}$, où pd représente la proportion de cas exposés au facteur de risque et RC représente le $\mathrm{RC}$ ajusté attribuable au facteur de risque ${ }^{19}$ ), ajusté pour l'âge maternel et le nombre d'accouchements antérieurs obtenus par des analyses distinctes des données pour les deux périodes indiquées.

Il a été démontré que le risque d'anomalies congénitales est de deux à cinq fois plus important en présence de diabète maternel qu'en l'absence de ce dernier ${ }^{7-11}$. La prévalence de diabète antérieur à la grossesse en Ontario a doublé entre 1996 et 2010 chez les femmes de 30 ans et plus $^{12}$, probablement en raison du mode de vie plus sédentaire et de la fréquence accrue de l'obésité. Entre 2000 et 2010, la prévalence du diabète dans l'ensemble de la population canadienne a augmenté de $103 \%$, pour atteindre 7,6 \%, avec des hausses particulièrement marquées chez les personnes de 35 à $44 a^{2} s^{23}$. En outre, on estime qu'environ $20 \%$ des cas de diabète ne sont pas diagnostiqués ${ }^{24}$. La d'un rapport de cotes de 2,1 (IC à $95 \%$ : 1,5 à 3,1 ) pour la prévalence des anomalies congénitales chez les nourrissons de femmes diabétiques ainsi que d'associations particulièrement robustes pour l'agénésie rénale, les anomalies obstructives du tractus urinaire, les cardiopathies congénitales et les anomalies congénitales multiples.

La maîtrise de la glycémie périconceptionnelle peut contribuer à réduire le risque d'anomalies congénitales associées au diabète $^{4,8,12,27}$. Dans une méta-analyse d'études d'observation portant sur la maîtrise de la glycémie périconceptionnelle, le risque d'anomalies congénitales augmentait avec chaque hausse de la concentration maternelle d'hémoglobine glyquée d'une unité d'écart-type selon un RC de 1,2 (IC à $95 \%: 1,1$ à 1,4$)^{27}$. Des études indiquent que les soins préconceptionnels sont associés à une concentration d'hémoglobine glyquée plus faible et à un risque d'anomalies congénitales significativement plus faible chez les femmes atteintes de diabète antérieur à la grossesse $^{13}$. Par conséquent, on devrait inclure des stratégies visant à renseigner les femmes diabétiques sur l'importance de soins de santé préconceptionnels et prénataux efficaces, particulièrement concernant la surveillance et la prise en charge de la glycémie, la pression artérielle et les complications liées aux organes cibles, par exemple les lésions de la rétine ou des reins $^{13,14,28,29}$

\section{Points forts et limites}

prévalence élevée tant de diabète que de surpoids et d'obésité antérieurs à la grossesse $^{25}$ est préoccupante, étant donné que ces facteurs sont significativement associés à un risque accru d'anomalies congénitales $^{25,26}$. En effet, les fœtus des mères obèses sont plus susceptibles d'être touchés par des anomalies du tube neural ou des anomalies cardiovasculaires que ceux des mères ayant un indice de masse corporel (IMC) normal ${ }^{25}$.

L'association que nous avons observée entre le diabète antérieur à la grossesse et les anomalies congénitales est cohérente avec d'autres études ${ }^{5-11}$. Par exemple, Nielsen et ses collaborateurs ${ }^{5}$ ont fait état
Notre étude comporte plusieurs limites. Premièrement, la diminution de la prévalence des anomalies congénitales pendant cette période est, en partie, causée par l'augmentation de l'accessibilité au dépistage prénatal et aux pratiques d'avortement en cas d'anomalies congénitales graves. Toutefois, notre source de données ne contenait pas de renseignements directs à propos du dépistage prénatal ou des pratiques de soins prénataux, ce qui nous a empêchés d'exclure ces effets. Depuis le milieu des années 1990, l'amélioration de la détection prénatale des anomalies congénitales peut avoir augmenté la fréquence des interruptions de grossesse de fœetus atteints d'anomalies congénitales graves, 
entraînant une diminution de la proportion de nourrissons vivants atteints d'anomalies congénitales ${ }^{2,30,31}$.

Deuxièmement, notre base de données associant les mères et les nourrissons incluait uniquement les naissances vivantes, donc sans mention des interruptions de grossesse tardives et des mortinaissances spontanées. Même si l'influence de l'inclusion des mortinaissances sur l'association entre le diabète et les anomalies congénitales n'est pas claire, il est probable que cette exclusion se traduise par une sous-estimation de l'association entre le diabète (particulièrement de type 1) maternel et les anomalies congénitales graves causées par cette affection. De plus, les anomalies congénitales étant diagnostiquées dans les 30 jours suivant la naissance, les anomalies détectées au cours de réadmissions plus tardives à l'hôpital n'ont pas été prises en compte. Il peut donc y avoir eu une sous-estimation de l'association entre diabète maternel et anomalies congénitales mineures ou certaines anomalies congénitales apparaissant plus tard au cours de l'enfance.

Troisièmement, aucune information sur les facteurs de risque maternels ou paternels d'anomalies congénitales autres que l'âge et le diabète maternels n'était accessible dans la BDCP. L'absence de ces données n'influence toutefois pas les tendances temporelles des anomalies congénitales examinées dans cette étude. En ce qui concerne la modification de la fréquence des anomalies du système nerveux central (principalement les anomalies congénitales du tube neural), l'enrichissement de la farine au moyen d'acide folique étant obligatoire au Canada depuis 1998, la diminution du nombre d'anomalies du tube neural s'est stabilisée avant le début de la période étudiée ${ }^{32,33}$. En outre, la légère augmentation des anomalies congénitales après 2007-2008 au Canada concorde avec l'augmentation du diabète antérieur à la grossesse et de l'âge maternel (figures 1 et 2). L'amélioration de la collecte de données dans une cohorte plus importante de femmes diabétiques avant leur grossesse, associée à un recensement détaillé des facteurs de risque (par exemple l'obésité maternelle), corrigerait certaines lacunes de notre étude ${ }^{1,31}$. Enfin, lorsque l'on sait que la mère est diabétique, on demande un diagnostic des anomalies congénitales, ce qui peut avoir entraîné un biais de détermination, particulièrement en ce qui concerne les anomalies congénitales mineures.

En résumé, même s’il y a une diminution relative de la prévalence à la naissance des anomalies congénitales au Canada, une plus grande proportion d'anomalies congénitales peut être attribuée à un diabète maternel antérieur à la grossesse. Ceci renforce l'importance à accorder aux programmes de soins préconceptionnels, particulièrement chez les femmes diabétiques, ainsi qu'à un dépistage prénatal précoce pour déceler les anomalies structurelles et orienter leur gestion. Les stratégies de traitement et de prévention du diabète chez les femmes en âge de procréer sont également importantes pour la prévention des anomalies congénitales causées par un diabète antérieur à la grossesse.

\section{Remerciements}

La présente étude a été réalisée avec l'appui du Système canadien de surveillance périnatale de l'Agence de la santé publique du Canada. Nous souhaitons remercier l'Institut canadien d'information sur la santé de nous avoir fourni l'accès à la Base de données sur les congés des patients (BDCP). Nous remercions également Susie Dzakpasu (Agence de la santé publique du Canada), Jane Evans (Université du Manitoba), Michael S. Kramer (Université McGill), Wei Luo, Chantal Nelson et Victoria Otterrman (tous les trois de l'Agence de la santé publique du Canada) pour leurs commentaires sur une version antérieure de cette étude. KSJ et JGR reçoivent le soutien d'une chaire de recherche appliquée décernée par les Instituts de recherche en santé du Canada.

\section{Références}

1. Agence de la santé publique du Canada. Anomalies congénitales au Canada 2013 : Rapport de surveillance sur la santé périnatale. Ottawa (Ont.) : Agence de la santé publique du Canada; 2013. PDF (2,09 Mo) téléchargeable à partir de la page: http://publications.gc. ca/site/fra/443925/publication.html.

2. Liu S, Joseph KS, Wen SW. Trends in fetal and infant deaths due to congenital anomalies. Semin Perinatol. 2002;26:268-276
3. Liu S, Joseph KS, Wen SW et collab. Secular trends in congenital anomaly-related fetal and infant mortality in Canada, 1985-1996. Am J Med Genet. 2001;104:7-13.

4. Jenkins KJ, Correa A, Feinstein JA et collab.; American Health Association Council on Cardiovascular Disease in the Young. Noninherited risk factors and congenital cardiovascular defects: current knowledge. A scientific statement from the American Heart Association Council on Cardiovascular Disease in the Young. Circulation. 2007;115:2995-3014.

5. Nielsen GL, Nogard B, Puho E, Rothman KJ, Sorensen HT, Czeizel AE. Risk of specific congenital abnormalities in offspring of women with diabetes. Diabet Med. 2005;22:693-696.

6. Liu S, Joseph KS, Lisonkova S et collab. Association between chronic medical conditions and congenital heart defects: a population-based cohort study. Circulation. 2013 128:583-9. DOI: 10.1161/CIRCULATIONAHA. 112.001054 .

7. Lisowski LA, Verheijen PM, Copel JA et collab. Congenital heart disease in pregnancies complicated by maternal diabetes mellitus: an international clinical collaboration, literature review, and metaanalysis. Herz. 2010;35:19-26.

8. Abu-Sulaiman RM, Subaih B. Congenital heart disease in infants of diabetic mothers: echocardiographic study. Pediatri Cardiol. 2004;25:137-140.

9. Wren C, Birrell G, Hawthorne G. Cardiovascular malformations in infants of diabetic mothers. Heart. 2003;89:1217-20.

10. Mikhail LN, Walker CK, Mittendorf R. Association between maternal obesity and fetal cardiac malformations in African Americans. J Natl Med Assoc. 2002;94695-700.

11. Fuhrmann K, Reiher H, Semmler K, Fischer F, Fischer M, Glockner E. Prevention of congenital malformations in infants of insulin-dependent diabetic mothers. Diabetes Care. 1983;6:219-223.

12. Feig DS, Hwee J, Shah BR, Booth GL, Bierman AS, Lipscombe LL. Trends in incidence of diabetes in pregnancy and serious perinatal outcomes: a large, population-based study in Ontario, Canada, 1996-2010. Diabetes Care. 2014;37: 1590-1596 
13. Wahabi HA, Alzeidan RA, Esmaeil SA. Pre-pregnancy care for women with pregestational diabetes mellitus: a systematic review and meta-analysis. BMC Public Health. 2012;12:792. DOI: 10.1186/14712458-12-792.

14. McLeod L, McLeod L, Ray JG. Prevention and detection of diabetic embryopathy. Community Genet. 2002;5:33-39.

15. Joseph KS, Fahey J pour le Système canadien de surveillance périnatale. Validation des données périnatales de la Base de données sur les congés des patients de l'Institut canadien d'information sur la santé. Maladies chroniques au Canada. 2009;29:96-100.

16. Liu S, Joseph KS, Hutcheon JA et collab. Gestational age-specific severe maternal morbidity associated with labor induction. Am J Obstet Gynecol. 2013;209. e1-e8.

17. Kramer MS, Rouleau J, Liu S, Bartholomew S, Joseph KS for the Maternal Health Study Group of the Canadian Perinatal Surveillance System. Amniotic fluid embolism: incidence, risk factors, and impact on perinatal outcome. BJOG. 2012;119: 874-879.

18. Agence de la santé publique du Canada. Rapport sur la santé périnatale au Canada, édition 2008. Ottawa (Ont.) : Ministère de la Santé; 2008. PDF (1,89 Mo) téléchargeable à partir de la page : http://www.phac-aspc.gc. ca/publicat/2008/cphr-rspc/pdf/cphr-rspc08fra.pdf

19. Rockhill B, Newman B, Weinberg C. Use and misuse of population attributable fractions. Am J Public Health. 1998;88:15-19.

20. Ferencz C, Loffredo CA, Correa-Villasenor A, Wilson PD (dir.). Genetic and environmental risk factors of major cardiovascular malformations: the Baltimore-Washington Infant Study 1981-1989. Armonk, NY : Futura Publishing Co.; 1997.

21. Czeizel AE. Periconceptional folic acid containing multivitamin supplementation. Eur J Obstet Gynecol Reprod Biol. 1998;78: 151-161.

22. Watkins ML, Botto LD. Maternal prepregnancy weight and congenital heart defects in offspring. Epidemiology. 2001;12:439-446.
23. Institut canadien d'information sur la santé (ICIS). Le moment propice : pourquoi l'âge de la mère est déterminant. ICIS; 2011. PDF (549 Ko) téléchargeable à partir du lien: https://secure.cihi.ca/free_products/ AIB InDueTime_WhyMaternalAgeMatters_F. pdf

24. Statistique Canada. Diabète, selon le groupe d'âge et le sexe (Pourcentage), tableau CANSIM 105-0501 [Internet]. Ottawa (Ont.) : Statistique Canada; [Modifié le 12 juin 2014; consultation le 6 août 2014]. Consultable en ligne à la page : http:// www.statcan.gc.ca/tables-tableaux/sum-som/ 102/cst01/health53b-fra.htm.

25. Stothard KJ, Tennant PW, Bell R, Rankin J. Maternal overweight and obesity and the risk of congenital anomalies: a systematic review and meta-analysis. JAMA. 2009;301:636-650. DOI: $10.1001 /$ jama.2009.113.

26. Mills JL, Troendle J, Conley MR, Carter T, Druschel CM. Maternal obesity and congenital heart defects: a population-based study. Am J Clin Nutr. 2010;91:1543-1549.

27. Gueirn A, Nisenbaum R, Ray JG. Use of maternal GHb concentration to estimate the risk of congenital anomalies in the offspring of women with prepregnancy diabetes. Diabetes Care. 2007;30:1920-1925.

28. Kitzmiller JL, Combs CA, Buchanan TA, Ratner RE, Kjos S. Pre-conception care of diabetes, congenital malformations, and spontaneous abortions. Diabetes Care. 1996;19:514-541.

29. Bialystok L, Poole N, Greaves L. Preconception care: call for national guidelines. Can Fam Physician. 2013;59:1037-1039.

30. Liu S, Joseph KS, Kramer MS et collab. Relationship of prenatal diagnosis and pregnancy termination to overall infant mortality in Canada. JAMA. 2002;287:15611567.

31. Joseph KS, Kinniburgh B, Hutcheon JA et collab. Determinants of increases in stillbirth rates from 2000 to 2010. CMAJ. 2013; 85(8):E345-51. DOI: 10.1503/cmaj.121372.

32. Bailey LB, Berry RJ. Folic acid supplementation and the occurrence of congenital heart defects, orofacial clefts, multiple births, and miscarriage. Am J Clin Nutr. 2005;81 (suppl):1213S-1237S.
33. De Wals P, Tairou F, Van Allen MI et collab. Reduction in neural-tube defects after folic acid fortification in Canada. N Engl J Med. 2007;357:135-142. 